\title{
DISTRIBUCIÓN DE LESIONES BUCALES PRODUCIDAS POR Candida albicans EN PACIENTES VIH/SIDA
}

Distribution of oral lesions produced by Candida albicans in HIV patients

\author{
Pedro Brevis ${ }^{1}$, César Coronado ${ }^{2}$, \\ Mario Cantin ${ }^{3}$, Paulina Abaca ${ }^{1}$ \\ 1 Departamento de Microbiología, Universidad de Talca, Chile. \\ 2 Unidad de Anatomía, Universidad de Talca, Chile \\ 3 Facultad de Odontología, Universidad de La Frontera, Temuco.
}

Correo electrónico:

Tel:

Palabras clave: Sindrome de Inmunodeficiencia Adquirida; Virus de Inmunideficiencia Humana; Candidiasis Bucal.

Key words: Acqired Immune Deficiency Syndrome; Human Immunodeficiency Virus; Oral Candidiasis.

\section{RESUMEN}

Antecedentes: La candidiasis orofaríngea es la infección fúngica oportunista mas frecuente en pacientes infectados por VIH. Clínicamente se puede presentar como queilitis angular, eritematosa, pseudomembranosa e hiperplásica.

Objetivo. En el presente estudio se analizó la distribución de lesiones candidiásicas y su ubicación en la cavidad bucal en 29 pacientes VIH positivo.

Método. Los pacientes fueron evaluados y controlados en el Servicio de Dermatología del Hospital Regional de Talca y se analizaron sus lesiones a nivel bucal y se tomaron muestras que permitieron aislar Candida albicans.

Resultados y conclusiones. La candidiasis pseudomembranosa fue encontrada en mayor porcentaje (47.1\%) y su principal ubicación fue en el paladar, la candidiasis eritematosa, la segunda más frecuente (45.1\%) y su principal ubicación fue el dorso de la lengua. La queilitis angular se presentó el menor porcentaje (7.8\%) y no se encontraron lesiones de tipo hiperplásica. Existe una fuerte asociación entre el tipo de variante clínica de candidiasis oral y su ubicación en la cavidad oral.

\section{INTRODUCCIÓN}

La candidiasis orofaríngea es la infección fúngica oportunista mas frecuente en pacientes infectados por el virus de la inmunodeficiencia humana (VIH). ${ }^{1}$ Se ha estimado que más del $90 \%$ de los pacientes infectados por

\begin{abstract}
Background. The oropharyngeal candidiasis is the most common opportunistic fungal infection in HIV- infected patients. Clinically can be presented as angular cheilitis, erythematous, pseudomembranous and hyperplastic .

Aims. The present study analyzed the clinical types of oral candidiasis and its location in the oral cavity in $29 \mathrm{HIV}$ positive patients

Methods.Patients were evaluated and controlled in a dermatology department of the Hospital Regional de Talca and analyzed their lesions at the oral level and samples for isolating Candida albicans.

Results and conclusions. Pseudomembranous candidiasis was found in highest percentage $(47.1 \%)$ and its main location was on the palate, erythematous candidiasis, the second most frequent $(45.1 \%)$ and its principal location was the back of the tongue. Angular cheilitis was presented the lowest percentage $(7.8 \%)$ and there were no hyperplastic type lesion. There is a strong association between the variant type of clinic oral candidiasis and its location in the oral cavity.
\end{abstract}

VIH pueden desarrollar esta infección en algún momento de la progresión de su enfermedad. ${ }^{2}$

$\mathrm{Su}$ agente causal en la mayoría de los casos corresponde a Candida albicans, ${ }^{1,3}$ cuya capacidad de persistir y causar la infección se ha atribuido a sus numerosas propiedades de virulencia, las cuales incluyen adhesión, dimorfismo, interferencia con el sistema inmunológico, 
sinergismo y la producción de enzimas extracelulares como proteinasas y lipasas. ${ }^{4} \mathrm{La}$ candidiasis orofaríngea es un marcador de inmunodeficiencia y ayuda al diagnóstico precoz de la infección por el VIH. ${ }^{5}$

Clínicamente se puede presentar como queilitis angular, candidiasis eritematosa, candidiasis pseudomembranosa y la hiperplásica o candidiasis crónica ${ }^{6,7}$ Las cuatro variantes clínicas ocurren con frecuencias distintas en pacientes con VIH-SIDA ${ }^{8}$

La candidiasis eritematosa aparecería de forma más precoz que la candidiasis pseudomembranosa que suele aparecer en pacientes con una inmunodeficiencia más grave. La queilitis angular y la candidiasis hiperplásica son las variantes menos comunes. ${ }^{9-12}$

Además, la candidiasis oral en la infección por VIH puede presentarse en múltiples localizaciones orales. Este fenómeno no es frecuente en personas VIH-negativas ${ }^{8}$, así, dos o los tres tipos de candidiasis oral (eritematosa, pseudomembranosa y queilitis angular) pueden aparecer juntas. ${ }^{13}$ El término candidiasis multifocal, es aplicado tanto para la presentación de una única variante clínica o diferentes variantes clínicas en dos o más localizaciones. ${ }^{14}$

En el presente estudio se analizó el tipo de lesiones candidiásicas presentes y la relación de éstas con su ubicación en la cavidad oral de pacientes VIH positivos controlados por el Servicio de Dermatología del Hospital Regional de Talca.

\section{MATERIALES Y MÉTODO}

Se realizó un estudio de candidiasis oral en 29 pacientes adultos de ambos sexos diagnosticados como seropositivos al VIH por el ISP (Instituto de Salud Pública), controlados en el Servicio de Dermatología del Hospital Regional de Talca, Chile. Todos los pacientes tenían signos y síntomas de candidiasis oral en algunas de sus manifestaciones clínicas. Los pacientes incorporados a este estudio fueron aquellos evaluados durante un período de cuatro meses.

Los pacientes fueron evaluados clínicamente por un mismo examinador en el Servicio de Dermatología del Hospital Regional de Talca. El material de exploración empleado para el examen intraoral consistió en un foco de luz artificial, guantes de látex, espejos intraorales y depresores linguales esterilizados. El examen se realizó tanto por observación indirecta como directa de las mucosas del paciente en donde se observaron: mucosas en relación al labio inferior y frenillo, mucosas en relación al labio superior y frenillos, reborde alveolar y encías, comisuras derecha e izquierda y zona retrocomisural, dorso y bordes laterales de lengua, cara ventral de lengua, piso de boca y paladar. Las lesiones analizadas fueron clasificadas mediante los siguientes criterios:

-Mucosa normal: coloración normal, sin signos de inflamación.

Candidiasis eritematosa: lesión de coloración rojiza en la mucosa, en la que pueden aparecer pequeñas placas o zonas puntiformes blanquecinas. La formación eritematosa destaca sobre la formación blanca.

Candidiasis pseudomembranosa: en forma de placas o nódulos blandos blanquecinos o amarillentos de aspecto cremoso que están dispuestos sobre una superficie mucosa ligeramente enrojecida. Se desprenden fácilmente al raspado.

Candidiasis hiperplásica: lesión blanca en forma de placas adheridas firmemente a la mucosa $o$ incluso nódulos exofíticos de aspecto blanquecino que no pueden ser desprendidas por el raspado.

Queilitis angular: lesiones generalmente bilaterales en las comisuras con pequeñas erosiones, fisuras o grietas que pueden presentar lesiones costrosas alrededor.

\section{Identificación de Candida albicans}

De cada paciente se obtuvieron muestras de las lesiones orales utilizando tórulas estériles previamente humedecidas con agua destilada estéril. Las muestras fueron procesadas en el Laboratorio de Micología de la Universidad de Talca.

La identificación de las levaduras a nivel de especie fue previamente comprobada según los procedimientos estándares empleados en micología médica, analizando las características fisiológicas, bioquímicas y micromorfológicas de cada cepa ${ }^{21}$.Las levaduras aisladas y mantenidas en discos de gelatina deshidratados fueron sembradas en tubos con agar Sabouraud-glucosa incubándolos en aerobiosis a $37^{\circ} \mathrm{C}$ por 24 a 48 horas. Posteriormente, se realizó la prueba fisiológica del tubo germinativo, para lo cual se inoculó, en un tubo estéril con $0,5 \mathrm{~mL}$ de plasma fresco humano, una asada del cultivo fresco de la levadura y se incubó por 2 a 3 horas a $37^{\circ} \mathrm{C}$. Luego con pipeta Pasteur se depositó una gota de esta suspensión en un portaobjeto, se cubrió con un cubreobjeto, y luego se 
examinó con aumento de 40x en el microscopio, en busca de prolongaciones finas de paredes paralelas, sin septos ni constricciones en la base de unión a la célula madre, siendo esta estructura característica de Candida albicans. Posteriormente, se efectuó un microcultivo para observar las características micromorfológicas de la levadura, tales como la presencia y forma de blastoconidios, clamidoconidios, pseudohifas. Para esto, se dispuso de una cámara húmeda estéril (placa Petri) compuesta de papel filtro, soporte y portaobjeto. Sobre este último se depositaron 2,5 a 3,0 $\mathrm{mL}$ de agar maíz con tween 80 al $1 \%$ previamente fundido a baño María. Luego de solidificado el medio, con un asa fina se realizó una siembra en estrías paralelas, en la superficie del medio. Para proteger los bordes de las estrías, se cubrió con un portaobjeto, siendo este el lugar de observación. Luego de humedecer la cámara con 2,5 a 3,0 mL de agua destilada estéril se incubó a $25^{\circ} \mathrm{C}$ por 48 a 72 horas, para luego ser examinado al microscopio con aumentos de 10x y 40x. Además se obtuvo un perfil bioquímico de cada cepa, comparando e interpolando los resultados obtenidos del auxonograma o asimilación de hidratos de carbono con las tablas de identificación respectiva ${ }^{21}$ Para esta prueba se preparó una suspensión de levadura a identificar, ajustando la turbidez de acuerdo al patrón 5 de la escala de MacFarland. Se fundió el medio C estabilizando su temperatura a $50^{\circ} \mathrm{C}$ en baño María. La proporción del inóculo versus medio de cultivo fue de 1:10, por lo que para verificar la asimilación de azúcares se mezcló $4 \mathrm{~mL}$ de la suspensión de levaduras con $40 \mathrm{~mL}$ del medio C y se traspasó a una placa Petri de $150 \mathrm{~mm}$ de diámetro., Luego de homogeneizar la mezcla se dejó solidificar un un lugar plano. Se distribuyeron los discos de carbohidratos sobre el medio C más levadura y se incubó a $25^{\circ} \mathrm{C}$ por 24 a 48 horas. La lectura se realizó observando un halo de crecimiento alrededor del disco. Los discos de azucares utilizados fueron: glucosa, galactosa, lactosa, maltosa, sacarosa, dulcitol, xilosa, melodiosa, rafinosa, manitol, inositol, L-arabinosa, celobiosa y trehalosa.

\section{RESULTADOS Y DISCUSIÓN}

Candida albicans se aisló en las lesiones bucales de los 29 pacientes.

De un total de 51 lesiones observadas en 29 pacientes, 23 correspondieron a candidiasis eritematosa, de las cuales 14 se ubicaron en el paladar, 8 en el dorso de la lengua y 1 en los bordes laterales de lengua; 24 correspondieron a candidiasis pseudomembranosa, de las cuales 3 se ubicaron en el paladar, 15 en el dorso de la lengua, 2 en los bordes laterales de lengua y 4 en otros; en la queilitis angular encontramos 4 muestras ubicadas en las comisuras labiales (Tabla 1).

La localización otros, corresponde a las ubicaciones menos frecuentes encontradas, correspondientes a cara interna de la mejilla, reborde alveolar y comisura. El análisis estadístico de los datos de la Tabla 1 con el Test Chi cuadrado $(\mathrm{P}=0,00)$ demostró una fuerte asociación entre el tipo clínico de candidiasis oral y la ubicación de estas en la cavidad oral.

\section{Discusión}

Los reportes de formas clínicas de candidiasis oral en pacientes VIH/SIDA difieren en cuanto a la forma de presentación predominante. Algunos autores como Martínez et al. ${ }^{12}$ y Flores et al. ${ }^{15}$ reportan que las lesiones pseudomembranosas, como ocurrió en este estudio $(47.1 \%)$ son las másfrecuentes.

Un estudio similar, realizado por Oliver et al. ${ }^{16}$ encontró también un predominio de esta variante con un $47,4 \%$, porcentaje muy similar al encontrado en este estudio. Se encontró a la variante eritematosa como la segunda más frecuente con 23 lesiones (45,1\%). Algunos como Félix \& Wray ${ }^{17}$ y Casariego \& Ben ${ }^{11}$ encuentran en su estudio mayor porcentaje de este tipo de lesiones. En un estudio realizado por Sharma et al. ${ }^{18}$ se encontró que la manifestación oral mas frecuente en pacientes infectados por VIH en India era la variante eritematosa. Solamente se encontraron 4 lesiones de queilitis angular (7.8\%), lo que concuerda con los reportes internacionales que aseguran que esta variante junto con la hiperplásica son las menos comunes ${ }^{8}$

La forma clínica de candidiasis oral hiperplásica no fue encontrada en este estudio, posiblemente debido al tamaño de la muestra, ya que la literatura señala porcentajes muy bajos de presentación de esta variante. ${ }^{9}$

Según nuestros resultados, existe una fuerte asociación entre el tipo de variante clínica de candidiasis oral y su ubicación en la cavidad oral, lo que podría ser de gran valor diagnóstico en caso es que la identificación de las lesiones tienda a confusión.

Las localizaciones más frecuentes de la candidiasis eritematosa según los reportes descritos por Bascones ${ }^{8} \mathrm{y}$ Delgado \& Aguirre ${ }^{19}$ son el paladar y el dorso de lengua. De las 23 lesiones de candidiasis eritematosa, 22 fueron encontradas en estas ubicaciones. El $61 \%$ se encontró en el paladar y el $34.8 \%$ en dorso de lengua, lo que corresponde a lo descrito por los autores antes mencionados. 
La candidiasis pseudomembranosa se localiza preferentemente según autores como Delgado \&Aguirre ${ }^{19}$ en la mucosa bucal, cara interna de labios y bordes laterales de lengua. En el actual trabajo, de las 24 lesiones encontradas, el $62.5 \%$, se ubicaron en el dorso de lengua, lo que difieren de la descrito en la literatura. Sin embargo, corresponde con lo encontrado por Samaranayake \& Holmstrup, ${ }^{20}$ él que en su estudio encontró que el mayor número de este tipo de lesiones se encontraban en el dorso de lengua. Además se encontró sólo 1 lesión en los bordes laterales de lengua, 3 lesiones en paladar, y 4 en otros lugares, esto no coinciden con lo descrito en la literatura, en cuanto a la ubicación preferentemente de este tipo de variante. Las cuatro lesiones de queilitis angular que se encontraron en este estudio se ubicaron en la comisura, lugar en que por definición se encuentra esta patología.

Se encontró que el sitio más frecuente afectado por lesiones en la cavidad oral fue la lengua, seguido por el paladar, existiendo una fuerte asociación entre el tipo de variante clínica de candidiasis oral y su ubicación en la cavidad oral, por lo que la ubicación depende del tipo de variante clínica de candidiasis.

\section{REFERENCIAS}

Rodríguez OJ, Miranda TJ, Morejon LH, Santana GJC. Candidiasis de la mucosa bucal: Revisión bibliográfica. Rev Cuba Estomatol 2002;39(2):187.233.

de Repentigny L, Lewandowski D, Jolicoeur P. Immunopathogenesis of oropharyngeal candidiasis in human immunodeficiency virus infection.Clin Microbiol Rev 2004;17(4): 729-59.

Menon T, Umamaheswari K, Kumarasamy N, Solomon S, Thyagarajan SP. Efficacy of fluconazole and itraconazole in the treatment of oral candidiasis in HIV patients. Acta Trop 2001; 80(2):151-4.

Willis AM, Couleter WA, Fulton CR Hayes JR, Bell PM, Lamet PJ. The influence of antifungal drugs or virulence properties of Candida albicans in patients with diabetes mellitus. Oral Surg Oral Med Oral Pathol Oral Radio Endod 2001;91(3):317-21.

Santamaría J, Sulibarria Z. Las micosis en los pacientes infectados porel VIH en la era de los tratamientos antirretrivirales de gran eficacia. Rev Iberoam Micol 2002;19:5-8.

Vultaggio A, Lombardelli L, Giudizi MG, Biagiotti R, Mazzinghi B, Scaletti C, et al.T cells specific for Candida albicans antigens and producing type 2 cytokines in lesional mucosa of untreated HIV-infected patients with pseudomembranous oropharyngeal candidiasis. Microbes Infect 2008;10(2):166-74.

Baccaglini L, Atkinson JC, Patton LL, Glick M, Ficarra G, Peterson DE. Management of oral lesions in HIV-positive patients. Oral Surg Oral Med Oral Pathol Radiol Endod 2007;103:1-23.

Bascones A. Candidosis orofaríngea, diagnóstico y tratamiento. Madrid: Ediciones avances, 1999.

Ramírez-Amador VA, Esquivel-Pedraza L, Ponce de León S, Ponce de León S. Prognostic value of oral candidosis and hairy leukoplakia in 111 Mexican HIV-infected patients. J Oral Pathol Med 1996;25(5):206-11.

Tsang PC, Samaranayake LP, Philipsen HIP, McCulloug M, Reichart PA, Schmidt- Westhausen A, et al. Biotypes of oral Candida albicans isolates in human immunodeficiency virus-infected patients from diverse geographic locations. J Oral Pathol Med 1995:24(1):32-6.

Casariego Z, Ben G.Manifestaciones bucales de la infección por VIH en Argentina. Estudio de 1889 casos. Medicina oral 1998;3:271-6.

Martínez M, Perurena L, Fernández A, Bandera T. Aislamiento, identificación y tipificación de levaduras en pacientes VIH positivos con candidiasis oral. Rev Cubana Med 1997;49(3):174-80.

Lopez-De-Blanc SA, Salati-De-Mugnolo N, Femopase FL, Benitez MB, Morelatto RA, Astrada-De-Verde L, Masih D. Antifungal topical therapy in oral chronic candidosis. A comparative study. Med Oral 2002;7(4):260-70.

Lazarde LJ, Añez OA. Candidiasis Multifocal bucal, reporte de un caso. Acta Odontol. Venezo. .2003;41(2):131-4.

Flores, Z.; Martínez, G.; Ruiz, A.; Fernández, C.;Mune,M.; Perurene, L. Candidiasis esofágica em pacientes com SIDA. 
Estúdio clínico y microbiológico. Ver Cubana Méd. Trop. 1998;50(2):110-114.

Oliver M, de González MI, Mendoza M, Bastardo de Albornoz MC. Serotypes of Candida albicans isolated from HIV positive patients. Rev. Iberoam. Micol. 1999;16(4):2047.

Félix D, Wray D. The prevalence of oral candidiasis in HIVinfected individuals and dental attenders in Edinburgh. J. Oral Pathol Med .1993;22(9):418-20.

Sharma G, Pai KM, Subas S, Ramapuram JT, Doshi D, Anup N. Oral manifestations in HIV/AIDS infected patients from Índia. Oral Dis 2006;12(6):537-42.

Delgado W, Aguirre J. Oral Mycoses in the AIDS era. Rev Iberoam Micol, 1997;14(1):14-22.

Samaranayeka LP, Holmstrup P. Oral candidiasis and human immunodeficiency virus infection. J Oral Pathol Med 1989;18(10):554-64.

Kurtzman C. \& Fell J. The yeast taxonomic study.4 Elseiver.1998 